for other risk factors. On average patients received $3.7 \pm 1.5$ injections, with 17,982 doses given overall. There were no drug related serious adverse events.296 infants required 357 hospitalizations for respiratory tract infections with a hospitalization rate of $6.0 \%$. There were significant differences between indications for palivizumab (chi-square=71.8, $\mathrm{p}<$ 0.005). The overall RSV positive HR was $1.38 \%$. Hospitalization rates were highest in infants of aboriginal descent $(15.0 \%$, chi-square $=22.2, \mathrm{p}<$ $0.005)$. Hospitalized infants had a lower percentage of compliant injections (62.8\% vs $68.6 \%, p=0.003)$.

Conclusions: The RSV HR in the 2006-2009 RSV seasons resembled several published reports (range $1.3 \%-5.3 \%$ ). RSV HR may be decreasing because of compliance with palivizumab prophylaxis, variability in RSV epidemiology, hospital admission criteria and preventive education.

1201

\section{RESPIRATORY SYNCYTIAL VIRUS PROPHYLAXIS IN SPECIAL POPULATIONS}

\author{
B. Paes ${ }^{1}$, A. Li ${ }^{2}$, K. Lanctot ${ }^{2}$, I. Mitchell ${ }^{3}$ \\ ${ }^{1}$ Pediatrics, McMaster University, Hamilton, \\ ${ }^{2}$ Medical Outcomes and Research in Economics \\ (MORE), Sunnybrook Health Sciences Centre, \\ Toronto, ON, ${ }^{3}$ Pediatrics, University of Calgary, \\ Calgary, AB, Canada
}

Objective: To determine palivizumab utilization in infants with pre-existing disease within the Canadian Registry Database (CARESS)

Methods: A prospective registry of infants from 27 sites who received palivizumab during the 2006-2009 RSV seasons. Demographic data were collected from the parent/caregiver at enrollment. Data on palivizumab utilization, compliance, and outcomes related to respiratory infection (RI) events were collected monthly. Infants $\leq 35$ weeks gestational age (GA) who met current approval criteria (Group 1) were compared to those with medical disorders who received off-label palivizumab (Group 2).

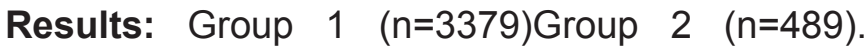
Male: $56.9 \%$ vs $54.9 \%(P=0.43)$. Average Enrollment Age (months) $\pm \mathrm{SD}: 3.6 \pm 3.4$ vs $9.9 \pm 8.8(\mathrm{P}=0.00)$. Average GA (weeks) Mean \pm SD: $31.0 \pm 3.1$ vs 37.1 $\pm 4.3(\mathrm{P}=0.00)$. Average \# injections $\pm \mathrm{SD}: 3.6 \pm 1.5$ vs $3.7 \pm 1.5(P=0.16)$. Hospitalization Rate $(H R)$ for Rl:4.1\% vs $9.2 \%$ ( $P=0.00$ ). RSV HR: $1.0 \%$ vs $2.2 \%$ $(\mathrm{P}=0.04)$.Compliant injections: $67.6 \%$ vs $67.6 \%$ $(P=0.99)$
Group 2 infants comprised Down syndrome $(n=118$, $24.1 \%)$, upper airway anomalies ( $n=112,22.9 \%)$, cystic fibrosis $(n=62,12.7 \%)$, neuromuscular impairment $(n=42,8.6 \%)$, pulmonary $(n=38,7.8 \%)$, multiple system disorders $(n=34,7.0 \%)$, cardiac $(n=17,3.5 \%)$, immunocompromise $(n=8,1.6 \%)$, and miscellaneous $(n=58,11.9 \%)$. The proportion of Group 2 infants receiving prophylaxis increased from 5.6\% (69/1224) to12.2\% (245/2016). Group 2 infants were older at enrollment with more advanced GA and had significantly higher RI and RSV hospitalization rates.

Conclusion: Clinicians strongly advocate for palivizumab in special populations based on perceived morbidity and mortality risk and evolving evidence from small cohort and case-controlled studies.

1202

\section{OUTCOMES OF BABIES WITH SEVERE RHESUS DISEASE FOLLOWING IN-UTERO TRANSFUSIONS - A 10 YEAR EXPERIENCE}

\author{
P. Chandra ${ }^{1}$, S.V. Rasiah ${ }^{1}$, A.K. Ewer ${ }^{1}$, M.D. Kilby ${ }^{2}$ \\ ${ }^{1}$ Neonatolgy, ${ }^{2}$ Maternal and Fetal Medicine, \\ Birmingham Women's Foundation NHS Trust, \\ Birmingham, UK
}

Background: In-utero transfusion (IUT) has transformed the management and outcome of severe Rhesus disease.

Objective: To assess the outcomes of babies that had IUT in a tertiary Fetal Medicine and red cell alloimmunization Centre.

Methods: Retrospective analysis of all cases of IUT for severe Rhesus disease over a 10 year period.

Results: 263 IUT were performed in 80 pregnancies. Median IUT was 3 (range 1-8). Antibodies involved were: anti-D (84\%); anti-Kell (13\%) and anti-c (3\%). $19(24 \%)$ cases were hydropic: of these 3 were intra-uterine deaths, 2 neonatal deaths and 3 were born elsewhere. 74 were live births (92.5\%); median gestation 34 weeks (range 24-38), 11 babies $\leq 30$ weeks, 5 born elsewhere. Of the 69 infants born in our centre, 3 died in the neonatal period and 1 infant at 19 months (SIDS). Median cord haemoglobin was $10.7 \mathrm{~g} / \mathrm{dL}$ (range 5.7-20.9). 29/69 (42\%) babies required 45 exchange transfusions (range 1-5). 57/69 (83\%) babies required 151 top-up blood transfusions (median 2, range 1-20). Of those $\leq 30$ weeks, 9/11 survived (82\%), 2 had retinopathy of prematurity and 
3 had cranial ultrasound abnormalities. At 2 years, $7 / 9$ babies were normal and 2 had developmental delay. 53/56 (95\%) surviving babies $>30$ weeks had normal neurodevelopment at 1 year except one with sensori-neural deafness.

Conclusion: IUT is safe and does not appear to increase postnatal morbidity. In our experience, it has reduced the need for postnatal exchange transfusions. However, top-up transfusions are more likely.

\section{3}

\section{CHARACTERISTICS AND OUTCOME OF CHILDREN REFERRED TO SPECIALIST PAEDIATRIC INTENSIVE CARE RERIEVAL TEAM WITH POISONING FROM DISTRICT GENERAL HOSPITAL}

\author{
M. Kumar, P. Ramnarayan \\ Great Ormond Street Hospital NHS Trust, \\ London, UK
}

Background: Childhood poisonings are common, but infrequently necessitate transfer to intensive care unit (ICU) for further management. This project was done to evaluate all the cases of poisoning referred to Children's Acute Transport Service (CATS), in terms of clinical chacteristics and outcome.

Method: All the children with acute poisoning for which advice or retrieval request was received at CATS, were included in this study. Various parameters noted were age, sex of child, type of poisoning/overdose agent, clinical manifestations, and management of child including any antidote used, reasons for refused referral, length of stay in PICU, final outcome of the episode and comparison of outcome of retrieved vs. not retrieved children.

Results: There were 42 cases of substance poisoning. Deliberate self harm was most common reason. Pharmaceuticals were most common agents involved.

Single agent was involved in most cases.

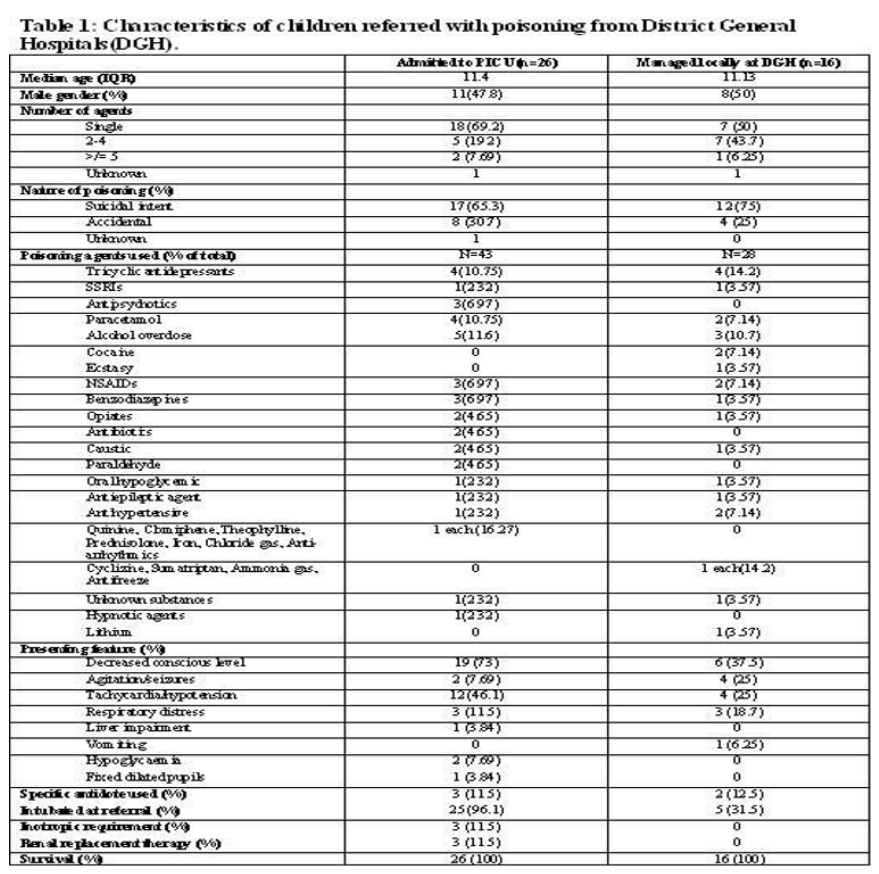

[Table 1. Characteristics of children.]

Nervous and cardiovascular symptoms were most common. Intensive supportive treatment was given in most of cases. In only 5 cases specific antidote was used. More than half didn't need retrieval because of improvement at referral hospital itself. No mortality was recorded.

Conclusions: Significant proportion of children referred for PICU can be managed at the local hospital, either with close observations and monitoring or temporary use of available adult critical care services for brief periods of ventilation. The vast majority of cases survive their acute episode following a relatively short spell of PICU, small minority may need advanced organ support, especially renal and cardiovascular.

\section{4}

\section{WHICH FLUID TO USE IN PEDIATRIC RESUSCITATION... STILL AN UNANSWERED QUESTION?}

\author{
M. Kumar ${ }^{1}$, S. Nadel ${ }^{2}$
}

${ }^{1}$ Pediatrics Intensive Care Unit, ${ }^{2}$ St. Mary's Hospital, Imperial College Health Care, NHS Trust, London, UK

Background and aims: The type of fluid used for resuscitation of patients with shock is subject to ongoing debate. This study was done to determine types of fluids used during resuscitation prior to admission to St Mary's PICU and to compare 